\title{
Discrete Magnetic Laplacian
}

\author{
M. A. Shubin ${ }^{\star}$ \\ Department of Mathematics, Northeastern University, Boston, MA 02115, USA, and Institute of \\ New Technologies, Kirovogradskaya, 11, Moscow 113587, Russia
}

Received: 26 July 1993/in revised form: 15 September 1993

\begin{abstract}
We consider a 2-dimensional discrete operator which we call the Discrete Magnetic Laplacian (DML); it is an analogue of the magnetic Schrödinger operator. It follows from well known arguments that DML has the same spectrum (as a subset in $\mathbf{R}$ ) as the Almost Mathieu operator (AM). They also have the same Integrated Density of States (IDS) which is known to be continuous. We show that DML is an element in a $\mathrm{II}_{1}$-factor and its IDS can be expressed through the trace in the $\mathrm{II}_{1}$-factor. It follows that DML never has any $L^{2}$-eigenfunctions (i.e. has no point spectrum). Then we formulate a natural algebraic conjecture which implies that the spectrum of DML (hence the spectrum of AM) is a Cantor set.
\end{abstract}

\section{Introduction}

Two main stars of this paper are the Discrete Magnetic Laplacian (DML) acting in $l^{2}\left(\mathbf{Z}^{2}\right)$ by the formula

$$
\begin{aligned}
\left(\Delta_{\alpha, \lambda} \psi\right)\left(n_{1}, n_{2}\right)= & e^{-i \pi \alpha n_{2}} \psi\left(n_{1}+1, n_{2}\right)+e^{i \pi \alpha n_{2}} \psi\left(n_{1}-1, n_{2}\right) \\
& +\lambda\left[e^{i \pi \alpha n_{1}} \psi\left(n_{1}, n_{2}+1\right)+e^{-i \pi \alpha n_{1}} \psi\left(n_{1}, n_{2}-1\right) ; n_{1}, n_{2} \in \mathbf{Z} ;\right.
\end{aligned}
$$

and the Almost Mathieu operator (AM) which acts in $l^{2}(\mathbf{Z})$ by the formula

$$
\left(H_{\alpha, \theta, \lambda} \psi\right)=\psi(n+1)+\psi(n-1)+2 \lambda \cos (2 \pi \alpha n+\theta) \psi(n) ; \quad n \in \mathbf{Z} .
$$

Here $\alpha, \lambda, \theta$ are real parameters.

The second operator (AM) was first introduced by R. Peierls [P] and has been extensively studied: an incomplete list of authors includes G. André, S. Aubry, J. Avron, Ya. Azbel, J. Bellissard, V. Buslaev, R. Carmona, W. Chambers, M.-D. Choi, V. Chulaevsky, F. Delyon, G. Elliott, A. Fedotov, A. Figotin, J. Fröhlich, P. Harper, B. Helffer, D. Hofstadter, S. Jitomirskaya (=Zhitomirskaya), P. Kerdelhué, Y. Last, R. Lima, V. Mandelshtam, P. van Mouche, L. Pastur, N. Riedel,

\footnotetext{
* Supported by NSF grant DMS-9222491
} 
B. Simon, Ya. Sinai, J. Sjöstrand, T. Spencer, Y. Tan, D. Testard, D. Thouless, G. Watson, M. Wilkinson, P. Wittwer, N. Yui (see e.g. [A-A, A-M-S, A-S, A, B1-B3, B-L-T1, B-L-T2, B-S, B-F, C-E-Y, C-D, D, C-F-K-S, E1, E2, F-P, F-S-W, Ha, H-K-S, H-S1-H-S3, Ho, J, L1, L2, L-W, M-Z, Mo, R1-R7, Si, T1, T2, T-T1, T-T2, $\mathrm{Wa}, \mathrm{W}]$ and references there). It has a physical meaning as a quantum Hamiltonian for a conduction electron in a magnetic field in a particular case of so-called tight-binding model ([A-A, A]). On the other hand more general difference Schrödinger operators with quasiperiodic potentials naturally appear when the perturbation technique is applied to a (continuous) magnetic Schrödinger operator in a homogeneous magnetic field with a small electric field ([N]).

The first operator (DML) is also well known though it appeared usually with $\lambda=1$ only, under different aliases and in a unitary equivalent form (see e.g. [Ha, Ho, B2]). It can also be considered as a Hamiltonian for a lattice electron in a magnetic field (then $\alpha$ corresponds to the magnetic flux through a unit cell). Much more general operators in $l^{2}\left(\mathbf{Z}^{2}\right)$ were introduced and studied by V.A. Mandelshtam and S.Ya. Zhitomirskaya [M-Z]. They established a connection between such operators and 1-dimensional discrete quasiperiodic operators. Due to some symmetries of the 2-dimensional operators this connection leads to a connection between spectra of different 1-dimensional quasiperiodic operators which is a generalization of the Aubry-Andre duality (see [A-A, A-S, F-P]). T. Sunada [Su] suggested and studied a generalization of DML to general graphs.

Note that in $[\mathrm{B} 2, \mathrm{Su}] \mathrm{DML}$ is called Harper operator. I prefer another name for it since the name Harper operator seems to be reserved for a one-dimensional difference operator on $\mathbf{R}$ (see e.g. [H-S1-H-S3, B-F]).

In 1981 during his talk in a session of American Mathematical Society Mark $\mathrm{Kac}$ offered ten Martinis to anybody who will prove that AM has Cantor spectra for any $\alpha \notin \mathbf{Q}$ (i.e. for any irrational $\alpha$ ) and any $\lambda \neq 0$. Being a witness of this talk B. Simon formulated this problem in his review paper [S] and labelled it as the Ten Martini Problem (TMP). In fact M. Kac might mean a stronger statement "all gaps are there" as quoted by B. Simon, who called it Strong (or Dry) form of TMP. (Let us abbreviate it STMP.) The exact meaning of this form is clear from the Gap Labelling Theory (GL) which was initiated by R. Johnson and J. Moser [J-M] and later developed connections with $C^{*}$-algebras and $K$-theory (see e.g. extensive review papers by $\mathrm{J}$. Bellissard [B1, B2] and references there). We will give more details about STMP later.

As far as I know TMP is still unsolved in spite of the announced counterexample [R5], though many partial results are known. For instance J. Bellissard and B. Simon [B-S] proved the M. Kac conjecture for a dense $G_{\delta}$ set of the pairs $(\alpha, \lambda)$ in $\mathbf{R}^{2}$; Ya. Sinai [Si] used KAM-theory to make it for almost all $\alpha$ (in fact all $\alpha$ that satisfy the Diophant condition of being badly approximated by rational numbers) but for large $\lambda$ only (it follows then from the Aubry-Andre duality [A-A, S] that the same is true for small $\lambda$ ); B. Helffer and J. Sjöstrand $[\mathrm{H}-\mathrm{S} 3]$, inspired by ideas of $\mathrm{M}$. Wilkinson [W], used the microlocal analysis to describe the tunneling between potential wells and solved TMP for the values of $\alpha$ which are presented by continuous fractions with big denominators; M.-D. Choi, G. Elliott and N. Yui [C-E-Y] used a beautiful algebraic technique to solve STMP in the case when $\alpha$ is a Liouville number (i.e. an irrational number which is exponentially good approximated by rational numbers). There is also strong numerical evidence in favor of the positive answer to TMP (see e.g. [Ho, H-K-S, B3]). 
In this paper we describe some ideas that might give an approach to TMP and STMP. This approach is based on studying DML instead of AM which is possible because their spectra coincide:

$$
\operatorname{spec}\left(H_{\alpha, \theta, \lambda}\right)=\operatorname{spec}\left(\Delta_{\alpha, \lambda}\right) \text { if } \alpha \notin \mathbf{Q} .
$$

This equality is well known (it follows e.g. from $[M-Z]$ in a much more general form). The simplest way to prove it is to observe (following e.g. arguments in [C-E-Y]) that both $H_{\alpha, \theta, \lambda}$ and $\Delta_{\alpha, \lambda}$ are images of the same element of the Rotation Algebra $A_{\alpha}$ (sometimes also called Irrational Rotation Algebra) under different representations. The algebra $A_{\alpha}$ is a remarkable $C^{*}$-algebra (a non-commutative 2-torus) which is defined by a particular case of a general crossed product construction (see Sect. 4 for a precise definition). Then (1.3) becomes an easy Corollary of the simplicity of $A_{\alpha}$ (see e.g. [E-H, Pe, Po, Z]).

An important spectral characteristic of almost periodic and random operators is their Integrated Density of States (IDS) - see e.g. [A-S, B1, B2, B-L-T2, C-L, C, C-F-K-S, J-M, P-F, S2, S3]. It plays a role of a spectrum distribution function and can be usually expressed in terms of a trace in a von Neumann algebra - see e.g. [B1, B2, B-L-T2, S2, S3]. In fact the expression in terms of the trace is often more convenient than the initial definition of IDS as a limit of normalized eigenvalue distribution functions over a family of blowing-up domains, and we prefer to start with the trace definition (as in [S1]).

We prove that $\Delta_{\alpha, \lambda}$ has a continuous IDS $N(\mu)$ which coincides with the IDS for $H_{\alpha, \theta, \lambda}$. But AM often has pure point spectrum with exponentially decaying eigenfunctions (this is the case e.g. if $\alpha$ has a Diophant property of being badly approximated by rationals and $\lambda$ is sufficiently large - see [Si, F-S-W]). On the other hand we prove that $\Delta_{\alpha, \lambda}$ never has any point spectrum (i.e. has no eigenvalues with eigenfunctions in $\left.l^{2}\left(\mathbf{Z}^{2}\right)\right)$. This follows easily from the fact that $\Delta_{\alpha, \lambda}$ is an element in a $\mathrm{II}_{1}$-factor $W_{\alpha}$ and its IDS is expressed in terms of the normalized trace $\tau$ on this factor

$$
N(\mu)=\tau\left(E_{\mu}\right),
$$

where $E_{\mu}$ is the spectral projection of $\Delta_{\alpha, \lambda}$. Actually we first introduce $N(\mu)$ using this formula as a definition. This allows us to make a shortcut to the proof of absence of point spectrum for $\Delta_{\alpha, \lambda}$, so any mentioning of AM and IDS becomes unnecessary.

Both the Rotation Algebra $A_{\alpha}$ and the $\mathrm{II}_{1}$-factor $W_{\alpha}$ can be considered as subalgebras in $\mathscr{B}\left(l^{2}\left(\mathbf{Z}^{2}\right)\right.$ ) (the algebra of all bounded linear operators in $l^{2}\left(\mathbf{Z}^{2}\right)$ ), $W_{\alpha}$ being the weak closure of $A_{\alpha}$. Denote also $C^{*}\left(\Delta_{\alpha, \lambda}\right)$ and $W^{*}\left(\Delta_{\alpha, \lambda}\right)$ the set of all continuous and bounded Borel functions of $\Delta_{\alpha, \lambda}$ respectively. They are commutative $C^{*}$ - and $W^{*}$-subalgebras in $A_{\alpha}$ and $W_{\alpha}$ respectively. Now our conjecture is that for any $\alpha \notin \mathbf{Q}$ and $\lambda \neq 0$,

$$
\left[U^{-1}\left(\operatorname{Proj}\left(A_{\alpha}\right)\right) U\right] \cap W^{*}\left(\Delta_{\alpha, \lambda}\right) \subset C^{*}\left(\Delta_{\alpha, \lambda}\right) \text { for any unitary } U \in W_{\alpha} .
$$

Here $\operatorname{Proj}\left(A_{\alpha}\right)$ is the set of all orthogonal projections in $A_{\alpha}$.

Using GL and simple von Neumann algebra arguments we show that (1.5) implies the positive answer to STMP. But (1.5) is a stronger statement so I will call it Superstrong Ten Martini Problem (SSTMP).

I tried to make this paper as self-contained as possible. This is the reason why some proofs are given that might be avoided if the economy would be properly 
pursued (e.g. the proof of continuity of the spectrum distribution function for DML given in Sect. 2 is not necessary since the fact may be easily established if we use the coincidence of this function with the IDS of AM as proved in Sect. 3 and then refer to the corresponding well-known fact for AM).

\section{2. $\mathrm{II}_{1}$-factor $W_{\alpha}$ and Absence of Point Spectrum for DML}

Let us introduce for any $\alpha \in \mathbf{R}$ unitary operators $U_{\alpha}, V_{\alpha}$ in $l^{2}\left(\mathbf{Z}^{2}\right)$ defined as follows:

$$
\left(U_{\alpha} \psi\right)\left(n_{1}, n_{2}\right)=e^{-i \pi \alpha n_{2}} \psi\left(n_{1}+1, n_{2}\right),\left(V_{\alpha} \psi\right)\left(n_{1}, n_{2}\right)=e^{i \pi \alpha n_{1}} \psi\left(n_{1}, n_{2}+1\right) .
$$

Here $n_{1}, n_{2} \in \mathbf{Z}$. Obviously $U_{\alpha} U_{\beta}=U_{\beta} U_{\alpha}, V_{\alpha} V_{\beta}=V_{\beta} V_{\alpha}$ for all $\alpha, \beta \in \mathbf{R}$. It is easy to check that

$$
U_{\alpha} V_{\beta}=e^{i \pi(\beta+\alpha)} V_{\beta} U_{\alpha} .
$$

In particular

$$
U_{\alpha} V_{\alpha}=e^{2 \pi i \alpha} V_{\alpha} U_{\alpha}
$$

and

$$
U_{\alpha} V_{-\alpha}=V_{-\alpha} U_{\alpha} .
$$

Consider in $l^{2}\left(\mathbf{Z}^{2}\right)$ the algebra $\mathscr{P}_{\alpha}$ of operators which can be represented as polynomials in $U_{\alpha}, V_{\alpha}, U_{\alpha}^{-1}, V_{\alpha}^{-1}$ :

$$
\mathscr{P}_{\alpha}=\left\{A \mid A=\sum_{|m|+|n| \leqq p} c_{m n} U_{\alpha}^{m} V_{\alpha}^{n} ; c_{m n} \in \mathbf{C}\right\} .
$$

(Here $p$ depends on $A$.) Obviously $\mathscr{P}_{\alpha}$ is a $*$-algebra, i.e. $B \in \mathscr{P}_{\alpha}$ implies $B^{*} \in \mathscr{P}_{\alpha}$.

We refer the reader to $[\mathrm{D} 1, \mathrm{D} 2, \mathrm{Mu}]$ for the necessary simplest definitions and facts about $C^{*}$-algebras and von Neumann algebras.

Denote $A_{\alpha}\left(\right.$ resp. $\left.W_{\alpha}\right)$ norm closure (resp. weak closure) of $\mathscr{P}_{\alpha}$. Hence

$$
\mathscr{P}_{\alpha} \subset A_{\alpha} \subset W_{\alpha},
$$

$A_{\alpha}$ is a $C^{*}$-algebra and $W_{\alpha}$ is a von Neumann algebra of operators in $l^{2}\left(\mathbf{Z}^{2}\right)$ (i.e. a weakly closed $*$-algebra containing the identity operator).

Now obviously

$$
\Delta_{\alpha, \lambda}=U_{\alpha}+U_{\alpha}^{*}+\lambda\left(V_{\alpha}+V_{\alpha}^{*}\right)=U_{\alpha}+U_{\alpha}^{-1}+\lambda\left(V_{\alpha}+V_{\alpha}^{-1}\right),
$$

hence $\Delta_{\alpha, \lambda} \in \mathscr{P}_{\alpha}$.

Note that $\Delta_{\alpha, \lambda}$ commutes with the operators $U_{-\alpha}, V_{-\alpha}$ which play a role similar to the role of the magnetic translations for a continuous magnetic Schrödinger operator.

We shall use the orthonormal basis of $\delta$-functions $\left\{\delta_{m} \mid m \in \mathbf{Z}^{2}\right\}$ in $l^{2}\left(\mathbf{Z}^{2}\right)$; here $\delta_{m}(n)=1$ if $m=n$ and 0 otherwise. If $A \in \mathscr{B}\left(l^{2}\left(\mathbf{Z}^{2}\right)\right)$ then we shall denote the matrix elements of $A$ in this basis by $A_{m, n}$, i.e. $A_{m, n}=\left(A \delta_{n}, \delta_{m}\right)$.

For subset $S \subset \mathscr{B}\left(l^{2}\left(\mathbf{Z}^{2}\right)\right)$ denote its commutant in $l^{2}\left(\mathbf{Z}^{2}\right)$ by $S^{\prime}$, i.e.

$$
S^{\prime}=\left\{B \mid B \in \mathscr{B}\left(l^{2}\left(\mathbf{Z}^{2}\right), \quad B A=A B \text { for every } A \in S\right\} .\right.
$$

Now we can describe the commutant of $W_{\alpha}$ and also give a description of $W_{\alpha}$ in terms of the matrix elements. 


\section{Proposition 2.1.}

(i) $W_{\alpha}^{\prime}=W_{-\alpha}$;

(ii) $W_{\alpha}$ coincides with the set of all $A \in \mathscr{B}\left(l^{2}\left(\mathbf{Z}^{2}\right)\right)$ such that

$$
A_{m, n} \exp (-i \pi \alpha m \wedge n)=A_{m+l, n+l} \exp (-i \pi \alpha(m+l) \wedge(n+l)), \quad m, n, l \in \mathbf{Z}^{2},
$$

where $m \wedge n=m_{1} n_{2}-m_{2} n_{1}$ if $m=\left(m_{1}, m_{2}\right), n=\left(n_{1}, n_{2}\right)$.

Proof. (a) Note first that (2.4) obviously implies that $W_{\alpha}^{\prime} \supset W_{-\alpha}$ (and also $\left.W_{-\alpha}^{\prime} \supset W_{\alpha}\right)$. Denote temporarily by $\tilde{W}_{\alpha}$ the set of all $A \in \mathscr{B}\left(l^{2}\left(\mathbf{Z}^{2}\right)\right)$ such that their matrix elements satisfy (2.7). We shall show now that $\tilde{W}_{\alpha}=W_{-\alpha}^{\prime}$. Clearly

$$
W_{-\alpha}^{\prime}=\left\{A \mid A \in \mathscr{B}\left(l^{2}\left(\mathbf{Z}^{2}\right)\right), U_{-\alpha}^{*} A U_{-\alpha}=A, V_{-\alpha}^{*} A V_{-\alpha}=A\right\} .
$$

Denote the canonical basis vectors in $\mathbf{Z}^{2}$ by $e_{1}, e_{2}: e_{1}=(1,0), e_{2}=(0,1)$. The matrix elements of the operator $A^{\prime}=U_{-\alpha}^{*} A U_{-\alpha}$ have the form

$$
\begin{aligned}
A_{m, n}^{\prime} & =\left(U_{-\alpha}^{*} A U_{-\alpha} \delta_{n}, \delta_{m}\right)=\left(A U_{-\alpha} \delta_{n}, U_{-\alpha} \delta_{m}\right)=\left(A e^{i \pi \alpha n_{2}} \delta_{n-e_{1}}, e^{i \pi \alpha m_{2}} \delta_{m-e_{1}}\right) \\
& =e^{i \pi \alpha\left(n_{2}-m_{2}\right)} A_{m-e_{1}, n-e_{1}}=e^{i \pi \alpha\left(m \wedge e_{1}+e_{1} \wedge n\right)} A_{m-e_{1}, n-e_{1}} .
\end{aligned}
$$

Hence

$$
e^{-i \pi \alpha\left(m-e_{1}\right) \wedge\left(n-e_{1}\right)} A_{m-e_{1}, n-e_{1}}=e^{-i \pi \alpha m \wedge n} A_{m, n}^{\prime},
$$

and $A^{\prime}=A$ is equivalent to (2.7) with $l=-e_{1}$. Replacing $m, n$ by $m+e_{1}, n+e_{1}$ respectively, we obtain (2.7) with $l=e_{1}$. Similarly $V_{-\alpha}^{*} A V_{-\alpha}=A$ is equivalent to (2.7) with $l=-e_{2}$ (or with $l=e_{2}$ ). But obviously the validity of (2.7) for all $m, n, l$ is equivalent to the validity of (2.7) for all $m, n$ and $l=e_{1}, e_{2}$. This proves that $W_{-\alpha}^{\prime}=\widetilde{W}_{\alpha}$ (and $W_{\alpha}^{\prime}=\tilde{W}_{-\alpha}$ since we can replace $\alpha$ by $-\alpha$ ). It follows in particular that $\tilde{W}_{\alpha} \supset W_{\alpha}$.

(b) Now let us check that $\tilde{W}_{\alpha} \subset \tilde{W}_{-\alpha}^{\prime}$, i.e. that $A \in \tilde{W}_{\alpha}, B \in \tilde{W}_{-\alpha}$ implies $A B=B A$. Note first that $A \in \tilde{W}_{\alpha}$ if and only if $A \in \mathscr{B}\left(l^{2}\left(\mathbf{Z}^{2}\right)\right)$ and

$$
A_{m, n} e^{-i \pi \alpha m \wedge n}=A_{0, n-m}, m, n \in \mathbf{Z}^{2} .
$$

Denote $a_{k}=A_{0, k}$, so $A_{m, n}=a_{n-m} e^{i \pi \alpha m \wedge n}$. Similarly for any $B \in \tilde{W}_{-\alpha}$ we have $B_{m, n}=b_{n-m} e^{-i \pi \alpha m \wedge n}$, where $b_{k}=B_{0, k}$.

Now denote $C^{(1)}=A B, C^{(2)}=B A$ and compare matrix elements of $C^{(1)}$ and $C^{(2)}$. We have

$$
C_{m, n}^{(1)}=\sum_{p \in \mathbf{Z}^{2}} A_{m, p} B_{p, n}=\sum_{p} a_{p-m} b_{n-p} e^{i \pi \alpha(m \wedge p-p \wedge n)}=\sum_{p} a_{p-m} b_{n-p} e^{i \pi \alpha(m+n) \wedge p}
$$

(this makes sense because $\left(a_{p}\right),\left(b_{p}\right) \in l^{2}\left(\mathbf{Z}^{2}\right)$ ). Similarly

$$
C_{m, n}^{(2)}=\sum_{q \in \mathbf{Z}^{2}} b_{q-m} a_{n-q} e^{-i \pi \alpha(m+n) \wedge q} .
$$

Replacing $q$ by $m+n-p$ here we get $C_{m, n}^{(2)}=C_{m, n}^{(1)}$ for all $m, n \in \mathbf{Z}^{2}$, hence $C^{(1)}=C^{(2)}$ as required.

(c) Due to the von Neumann Double Commutant Theorem (see e.g. [D1, Mu]) we have $W_{\alpha}=W_{\alpha}^{\prime \prime}=\tilde{W}_{-\alpha}^{\prime}$. It follows that

$$
W_{\alpha} \subset \tilde{W}_{\alpha}=W_{-\alpha}^{\prime} \subset \tilde{W}_{-\alpha}^{\prime}=W_{\alpha},
$$

hence $W_{\alpha}=\tilde{W}_{\alpha}=W_{-\alpha}^{\prime}$ which proves the proposition. 
Now the next step will be to describe the center $Z\left(W_{\alpha}\right)=W_{\alpha} \cap W_{\alpha}^{\prime}$ of the von Neumann algebra $W_{\alpha}$. It can be easily done for any $\alpha$, but we will do it for the simplest case of an irrational $\alpha$ only.

Proposition 2.2. If $\alpha \notin \mathbf{Q}$ then the center $Z\left(W_{\alpha}\right)$ is trivial, i.e. $Z\left(W_{\alpha}\right)=\{\lambda I \mid \lambda \in \mathbf{C}\}$, where $I$ is the identity operator. In other words $\alpha \notin \mathbf{Q}$ implies that $W_{\alpha}$ is a factor.

Proof. Obviously $Z\left(W_{\alpha}\right)$ is the set of all operators $A$ in $l^{2}\left(\mathbf{Z}^{2}\right)$ that commute with $U_{\alpha}, U_{-\alpha}, V_{\alpha}, V_{-\alpha}$. But then $A$ commutes with $M_{0,1}=U_{\alpha}^{*} U_{-\alpha}$ and $M_{1,0}=V_{-\alpha}^{*} V_{\alpha}$ which are multiplication operators by $\exp \left(2 \pi i \alpha n_{1}\right)$ and $\exp \left(2 \pi i \alpha n_{2}\right)$ respectively. It follows that $A$ commutes with any multiplication operator $M_{k}=M_{1,0}^{k_{1}} M_{0,1}^{k_{2}}$, where $k=\left(k_{1}, k_{2}\right) \in \mathbf{Z}^{2}$. This operator acts as follows:

$$
\left(M_{k} \psi\right)(n)=e^{2 \pi i \alpha k \cdot n} \psi(n), \quad \psi \in l^{2}\left(\mathbf{Z}^{2}\right),
$$

where $k \cdot n=k_{1} n_{1}+k_{2} n_{2}$. Now if $A_{m, n}$ are the matrix elements of $A$ then the operator $M_{k}^{-1} A M_{k}$ has matrix elements $A_{m, n} \exp (-2 \pi i \alpha k \cdot(m-n))$. Their coincidence with $A_{m, n}$ for all $k$ obviously implies that $A_{m, n}=0$ if $m \neq n$, since for any $m, n \in \mathbf{Z}^{2}$ with $m \neq n$ we can always find $k \in \mathbf{Z}^{2}$ such that $\exp (-2 \pi i \alpha k \cdot(m-n)) \neq 1$. Therefore if $A \in Z\left(W_{\alpha}\right)$, then $A_{m, n}=a_{m} \delta_{m}(n), a_{m} \in \mathbf{C}$.

Now consider the translation operators $T_{k}, k \in \mathbf{Z}^{2}$, acting as follows:

$$
T_{k} \psi(n)=\psi(n+k), \psi \in l^{2}\left(\mathbf{Z}^{2}\right) .
$$

The operators $T_{1,0}, T_{0,1}$ are compositions of $U_{\alpha}, V_{\alpha}$ with multiplication operators. Hence $A \in Z\left(W_{\alpha}\right)$ implies that $A$ commutes with all $T_{k}$. It follows that $A_{m+l, n+l}=A_{m, n}$ for all $m, n, l \in \mathbf{Z}^{2}$. Hence $A_{m, n}=a \delta_{m}(n), a \in \mathbf{C}$, and the Proposition follows.

Now we shall introduce the trace on $W_{\alpha}$. Define for any $A \in \mathscr{B}\left(l^{2}\left(\mathbf{Z}^{2}\right)\right)$

$$
\tau(A)=A_{0,0}=\left(A \delta_{0}, \delta_{0}\right) .
$$

So $\tau$ is a linear map from $\mathscr{B}\left(l^{2}\left(\mathbf{Z}^{2}\right)\right)$ to $\mathbf{C}$. Actually we shall only use the restriction of $\tau$ to $W_{\alpha}$ for a fixed $\alpha$. We shall also denote this restriction by $\tau$. Note that (2.8) implies that $A_{m, m}=A_{0,0}$ for any $m \in \mathbf{Z}^{2}$ and any $A \in W_{\alpha}$.

Proposition 2.3. $\tau$ is a weakly continuous trace on $W_{\alpha}$. More exactly $\tau$ is a weakly continuous linear function on $W_{\alpha}$ satisfying the following conditions:

(i) $\tau(A B)=\tau(B A), A, B \in W_{\alpha}$;

(ii) $A \geqq 0$ implies $\tau(A) \geqq 0$ with the equality for $A=0$ only;

(iii) $\tau(I)=1$.

Proof. Note first that (iii) and the first part of (ii) are obvious. Now $A \geqq 0$ and $\tau(A)=0$ imply $A_{m, m}=0$ for all $m \in \mathbf{Z}^{2}$, hence $A_{m, n}=0$ for all $m, n \in \mathbf{Z}^{2}$ because of the Cauchy-Schwarz inequality $\left|A_{m, n}\right|^{2} \leqq A_{m, m} A_{n, n}$. So the second part of (ii) follows.

To check (i) note that in terms of the matrix elements $A_{m, n}$ and $B_{m, n}$ of the operators $A$ and $B$ we have

$$
\tau(A B)=\sum_{m \in \mathbf{Z}^{2}} A_{0, m} B_{m, 0}=\sum_{m \in \mathbf{Z}^{2}} A_{-m, 0} B_{0,-m}=\sum_{m \in \mathbf{Z}^{2}} B_{0, m} A_{m, 0}=\tau(B A),
$$

since $A_{0, m}=A_{-m, 0}, B_{m, 0}=B_{0,-m}$ due to (2.7). 
Propositions 2.2 and 2.3 imply that $W_{\alpha}$ is a $\mathrm{II}_{1}$-factor for any $\alpha \notin \mathbf{Q}$. It follows that the trace $\tau$, satisfying conditions of Proposition 2.3 is unique for irrational $\alpha$.

Now we can use the trace to define the spectrum distribution function for a self-adjoint operator in $W_{\alpha}$.

Let $A=A^{*} \in W_{\alpha}, A=\int \mu d E_{\mu}$ be the spectral decomposition of $A$, so $E_{\mu}^{2}=E_{\mu}=E_{\mu}^{*}, E_{\mu} \geqq E_{\mu^{\prime}}$ if $\mu \geqq \mu^{\prime}, E_{\mu+0}=E_{\mu}, E_{\mu}=0$ if $\mu<-\|A\|, E_{\mu}=I$ if $\mu>\|A\|$. Note that $A \in W_{\alpha}$ if and only if $E_{\mu} \in W_{\alpha}$ for all $\mu \in \mathbf{R}$.

Definition 2.4. The function

$$
N(\mu ; A)=\tau\left(E_{\mu}\right)
$$

is called the spectrum distribution function (SDF) of the self-adjoint operator $A \in W_{\alpha}$. We will also write simply $N(\mu)$ for brevity's sake if it is clear which operator $A$ is taken.

Let $\operatorname{spec}(A)$ denotes the spectrum of $A$. Then obviously

$$
\operatorname{spec}(A)=\{\mu \mid N(\mu+\varepsilon ; A)-N(\mu-\varepsilon ; A)>0 \text { for any } \varepsilon>0\},
$$

i.e. $\sigma(A)$ is the set of all points of growth of SDF.

Later in this paper we will show that SDF for the DML $\Delta_{\alpha, \lambda}$ coincides with the IDS of this operator. But actually we do not need IDS for our purposes.

Denote

$$
E_{\{\mu\}}=E_{\mu+0}-E_{\mu-0},
$$

i.e. $E_{\{\mu\}}$ is the projection on the eigenspace $\operatorname{Ker}(A-\mu I)$ of $A$ with the eigenvalue $\mu$.

Lemma 2.5. $\tau\left(E_{\{\mu\}}\right)=N(\mu+0)-N(\mu-0)$,

i.e. $\tau\left(E_{\{\mu\}}\right)$ is equal to the jump of SDF at $\mu$.

Proof. is obvious due to the weak continuity of $\tau$.

Corollary 2.6. $\mu \in \mathbf{R}$ is in the point spectrum of $A$ (i.e. $\operatorname{Ker}(A-\mu I) \neq\{0\})$ in $l^{2}\left(\mathbf{Z}^{2}\right)$ if and only if SDF of $A$ is discontinuous (hence has a jump) at $\mu$. In particular, SDF of $A$ is continuous if and only if $A$ has no point spectrum.

Note that Definition 2.4, Lemma 2.5 and Corollary 2.6 are all valid in a general abstract context for any von Neumann algebra with a faithful finite trace (in particular for any $\mathrm{II}_{1}$-factor) and any self-adjoint operator in this algebra.

Theorem 2.7. SDF for $\Delta_{\alpha, \lambda}$ is continuous or, equivalently, $\Delta_{\alpha, \lambda}$ has no point spectrum.

Proof. To prove the continuity of the IDS for the DML it is sufficient to repeat the argument given by F. Deylon and B. Souillard [D-S] (see also the book [C-F-K-S]) for discrete Schrödinger operators. Results of Sect. 3 provide another proof of Theorem 2.7 based on the fact that the SDF for $\Delta_{\alpha, \lambda}$ coincides with the IDS of a one-dimensional discrete Schrödinger operator (which is the Almost Mathieu operator). In particular this will imply the log Hölder continuity of the SDF due to the result of W. Craig and B. Simon [C-S] for discrete Schrödinger operators. 


\section{Integrated Density of States}

Let us fix $T \geqq 1$ and consider a family $\mathscr{F}$ of finite subsets $\Lambda \subset \mathbf{Z}^{2}$ and a family of self-adjoint operators $H_{A}$ in $l^{2}(\Lambda)$ such that the following two conditions are satisfied:

(a) $H_{\Lambda} \psi(x)=\Delta_{\alpha, \lambda} \psi(x)$ if $x \in \Lambda$ and $\operatorname{dist}\left(x, \Lambda^{c}\right)>T ; \psi \in l^{2}(\Lambda)$;

(Here dist means the standard euclidean distance and $\Lambda^{c}=\mathbf{Z}^{2}-\Lambda$ is the complement of $\Lambda$ in $\mathbf{Z}^{2}$.)

(b) $\left\|H_{\Lambda}\right\| \leqq C$, where $C$ does not depend on $\Lambda \in \mathscr{F}$.

An example of such a family: $H_{A}=\chi_{A} H_{A} \chi_{\Lambda}$, where $\chi_{\Lambda}$ is the characteristic function of $\Lambda$; this means that we impose the Dirichlet boundary conditions on the "boundary" of $\Lambda$.

Now denote for any $T \geqq 1$,

$$
(\partial \Lambda)_{T}=\left\{x \mid x \in \Lambda, \operatorname{dist}\left(x, \Lambda^{c}\right) \leqq T\right\} .
$$

For any finite set $K$ let $|K|$ denote the number of points in $K$. Our next definition will describe the situation when $\Lambda$ blows up in a reasonable way.

Definition 3.1. We shall write $\Lambda \rightarrow \infty$ if for any fixed $T \geqq 1$,

$$
\frac{\left|(\partial \Lambda)_{T}\right|}{|\Lambda|} \rightarrow 0 \text {. }
$$

(It is understood here that $\Lambda$ runs through a sequence or an directed set of finite subsets in $\mathbf{Z}^{2}$; the direction can be given in an abstract way and not necessarily by inclusion.)

Denote by $N_{A}$ the standard distribution function of eigenvalues for $H_{A}$ (so $N_{\Lambda}(\mu)$ is the number of eigenvalues $\leqq \mu$, multiplicities counted). The normalized function $|\Lambda|^{-1} N_{\Lambda}$ may only have values of the form $|\Lambda|^{-1} j, j=0,1,2, \ldots,|\Lambda|$.

Theorem 3.2. If $H_{\Lambda}$ satisfies (a), (b), then for all $\mu \in \mathbf{R}$,

$$
\lim _{\Lambda \rightarrow \infty}|\Lambda|^{-1} N_{\Lambda}(\mu)=N\left(\mu ; \Delta_{\alpha, \lambda}\right) .
$$

The left-hand side of this formula is called the Integrated Density of States (IDS) for DML. It follows that the limit does not depend on the chosen family $\left\{H_{A}\right\}$ and can be expressed in terms of the trace in a $\mathrm{II}_{1}$-factor.

The existence of the limit similar to the left-hand side of (3.2) was proved for the first time by I. Slivnyak [SI] (he considered continuous Schrödinger operators with random ergodic potentials). Later this result was modified, extended and given a different proof in papers of L. Pastur (see e.g. [Pa, P-F] and references there). The case of higher order differential operators with almost periodic and random coefficients was considered in papers by M. Shubin (see e.g. [S2, S3]) and A. Gusev [G] (the paper [G] relied on ideas and methods developed in [S2] and was submitted later, though published earlier, than [S2]). Note however that the formula (3.2) itself does not follow from the general theory of ergodic operators. For the first time a formula of this type was established in [S2] (for general elliptic operators with almost periodic coefficients). About other situations where such a result is true see [B1, B2, B-L-T2] and references there. 
The proof of the theorem will use the main idea of the corresponding proof in [S2] but will follow closely its realization in [B-L-T2] where useful modifications are made. We will start with some straightforward lemmas (the proofs of them are left to the reader). As before we denote the matrix elements of a bounded linear operator $A: l^{2}\left(\mathbf{Z}^{2}\right) \rightarrow l^{2}\left(\mathbf{Z}^{2}\right)$ in the standard $\delta$-function basis by $A_{m, n}, m, n \in \mathbf{Z}^{2}$.

\section{Lemma 3.3.}

$$
\left(H_{\Lambda}^{k}\right)_{m, n}=\left(\Delta_{\alpha, \lambda}^{k}\right)_{m, n} \text { if } m, n \in \Lambda, k \in \mathbf{Z}_{+} \text {and } \operatorname{dist}\left(m, \Lambda^{c}\right)>k T .
$$

Lemma 3.4. For every $t \in \mathbf{C}$,

$$
\left.\mid\left(e^{t H} \Lambda\right)\right)_{m, n}-\left(e^{t \Delta_{\alpha, \lambda}}\right)_{m, n} \mid \leqq f\left(\operatorname{dist}\left(m, \Lambda^{c}\right)\right), m, n \in \Lambda,
$$

where

$$
f(s)=\sum_{k \geqq s / T} \frac{|t|^{k}(C+4)^{k}}{k !},
$$

so that $f=f_{i}: \mathbf{R}_{+} \rightarrow \mathbf{R}_{+}$is bounded and $f(s) \rightarrow 0$ as $s \rightarrow \infty$. Here $C$ is the constant from the condition (b).

Lemma 3.5. If $f: \mathbf{R}_{+} \rightarrow \mathbf{R}_{+}$is bounded and $f(s) \rightarrow 0$ as $s \rightarrow \infty$, then

$$
\lim _{\Lambda \rightarrow \infty} \frac{1}{|\Lambda|} \sum_{m \in \Lambda} f\left(\operatorname{dist}\left(m, \Lambda^{c}\right)\right)=0 \text {. }
$$

Proof of Theorem 3.2. Lemmas 3.4 and 3.5 imply that

$$
\lim _{\Lambda \rightarrow \infty} \frac{1}{|\Lambda|} \sum_{m \in \Lambda}\left[\left(e^{t H_{\Lambda}}\right)_{m, m}-\left(e^{t \Delta_{\alpha}, \Lambda}\right)_{m, m}\right]=0 .
$$

Note that

$$
\left(e^{t \Delta_{\alpha, \lambda}}\right)_{m, m}=\left(e^{t \Delta_{\alpha, \lambda}}\right)_{0,0}=\tau\left(\left(e^{t \Delta_{\alpha}, \lambda}\right)\right),
$$

since $\Delta_{\alpha, \lambda} \in W_{\alpha}$. Therefore

$$
\lim _{\Lambda \rightarrow \infty} \frac{1}{|\Lambda|} \sum_{m \in \Lambda} \operatorname{Tr} e^{t H_{\Lambda}}=\tau\left(e^{t \Delta_{\alpha \prime \prime}}\right) .
$$

But $|\Lambda|^{-1} \sum_{m \in \Lambda} \operatorname{Tr} e^{t H_{\Lambda}}$ and $\tau\left(e^{t \Delta_{\alpha}, \lambda}\right)$ are the Laplace transforms of measures $|\Lambda|^{-1} d N_{\Lambda}(\mu)$ and $d N\left(\mu ; \Delta_{\alpha, \lambda}\right)$, i.e.

$$
|\Lambda|^{-1} \operatorname{Tr} e^{t H_{\Lambda}}=|\Lambda|^{-1} \int_{\mathbf{R}} e^{t \mu} d N_{\Lambda}(\mu), \quad \tau\left(e^{t \Delta_{\alpha, \lambda}}\right)=\int_{\mathbf{R}} e^{t \mu} d N\left(\mu ; \Delta_{\alpha, \lambda}\right) .
$$

Since all the functions $|\Lambda|^{-1} N_{\Lambda}(\mu)$ are uniformly bounded (by 1), the well known weak compactness results imply (3.2) (see e.g. [S3], Lemma 2.1) which proves the Theorem.

\section{Spectrum and Integrated Density of States for AM}

$A$. Now we turn to the IDS for the AM $H_{\alpha, \theta, \lambda}$ (see (1.2)). Here the existence of IDS and its expression in terms of the trace in factors of type II is well known (see e.g. [A-S, B-L-T2, C-L, C, C-F-K-S, P-F, S2, S3]) but for the sake of completeness we 
shall repeat the arguments and slightly modify them to achieve a bigger generality. Actually almost no changes are necessary comparing with the case of DML. So let us fix $T \geqq 1$ and consider a family $\mathscr{F}$ of finite sets $\Lambda \subset \mathbf{Z}$ and a family of self-adjoint operators $H_{\Lambda}$ in $l^{2}(\Lambda)$ such that

(a') $H_{\Lambda} \psi(x)=H_{\alpha, \theta, \lambda} \psi(x)$ if $x \in \Lambda$ and $\operatorname{dist}\left(x, \Lambda^{c}\right)>T ; \psi \in l^{2}(\Lambda)$;

(b') $\left\|H_{\Lambda}\right\| \leqq C$, where $C$ does not depend on $\Lambda \in \mathscr{F}$.

The notation $(\partial \Lambda)_{T}$ and the Definition 3.1 obviously apply to this case. The usually considered family $\mathscr{F}$ is the set of intervals $[a, b] \cap \mathbf{Z}$ and $A \rightarrow \infty$ means that $b-a \rightarrow \infty$.

Now denote the eigenvalue distribution function of $H_{\Lambda}$ by $N_{\Lambda}$.

Theorem 4.1. If $H_{\Lambda}$ satisfies $\left(\mathrm{a}^{\prime}\right),\left(\mathrm{b}^{\prime}\right)$ then for all $\mu \in \mathbf{R}$ there exists a limit

$$
N_{1}\left(\mu ; H_{\alpha, \theta, \lambda}\right)=\lim _{\Lambda \rightarrow \infty}|\Lambda|^{-1} N_{\Lambda}(\mu),
$$

which does not depend on the choice of the family $\left\{H_{\Lambda} \mid \Lambda \in \mathscr{F}\right\}$ and is called IDS for $H_{\alpha, \theta, \lambda}$.

Moreover, the Laplace transform of the measure corresponding to the IDS is given by the formula

$$
\int e^{t \mu} d N_{1}\left(\mu ; H_{\alpha, \theta, \lambda}\right)=M_{m}\left\{\left(e^{t H_{\alpha, \theta, \lambda}}\right)_{m, m}\right\},
$$

where $M_{m}$ means the mean value of a Bohr almost periodic function on $\mathbf{Z}$ with respect to $m$ :

$$
\left.M_{m}\{f(m))\right\}=\lim _{N \rightarrow \infty} \frac{1}{2 N+1} \sum_{-N \leqq m \leqq N} f(m) .
$$

Proof. Lemmas 3.3, 3.4 and 3.5 are true in this case (with $\Delta_{\alpha, \lambda}$ replaced by $H_{\alpha, \theta, \lambda}$ ), literally the same proofs apply. Therefore we come to the equality

$$
\lim _{\Lambda \rightarrow \infty} \frac{1}{|\Lambda|} \sum_{m \in \Lambda}\left[\left(e^{t H_{\Lambda}}\right)_{m, m}-\left(e^{t H_{\alpha, \theta, \gamma}}\right)_{m, m}\right]=0 .
$$

It remains to notice that $m \mapsto\left(e^{t H_{\alpha, \theta, \lambda}}\right)_{m, m}$ is a Bohr almost periodic function since this is true for all functions $m \mapsto\left(H_{\alpha, \theta, \lambda}^{k}\right)_{m, m}, k=0,1,2, \ldots$ The existence of the mean value for a Bohr almost periodic function along a family of $\Lambda$ satisfying the condition (3.1), is easy to check as in the continuous case (see e.g. [S2]) or just by noticing that it is sufficient to consider the case of the exponents $m \mapsto e^{i \xi \cdot m}$ for all $\xi \in \mathbf{R}$, where this existence is obvious.

$B$. We shall need the Rotation Algebra in the abstract form: this is the $C^{*}$-algebra generated by two unitary elements $u, v$ with the commutation relation

$$
u v=e^{2 \pi i \alpha} v u \text {. }
$$

First consider the algebra $\mathscr{P}_{\alpha}$ of all Laurent polynomials

$$
\mathscr{P}_{\alpha}=\left\{a \mid a=\sum_{|k|,|l|, \leqq N} c_{k l} u^{k} v^{l}, \quad N \in \mathbf{Z}_{+}, c_{k l} \in \mathbf{C}\right\}
$$

where the multiplication is naturally defined with the use of (4.3). It has a unique antilinear involution such that $1^{*}=1, u^{*}=u^{-1}, v^{*}=v^{-1}$. A representation of this 
algebra is a $*$-homomorphism $\pi: \mathscr{P}_{\alpha} \rightarrow \mathscr{B}(\mathscr{H})$, where $\mathscr{H}$ is a Hilbert space. Such representations are in one-one correspondence with couples $U, V$ of unitary operators (in $\mathscr{H}$ ) such that (4.3) is true with $u, v$ replaced by $U, V$. Now the $C^{*}$-norm on $\mathscr{P}_{\alpha}$ is defined as

$$
\|a\|=\sup _{\pi}\|\pi(a)\|
$$

where $\|\pi(a)\|$ is the operator norm of $\pi(a)$, supremum is taken over all representations of $\mathscr{P}_{\alpha}$ in Hilbert spaces.

Obviously

$$
\left\|\sum_{k, l} c_{k, l} u^{k} v^{l}\right\| \leqq \sum_{k, l}\left|c_{k, l}\right|,
$$

hence the norm (4.4) is finite on $\mathscr{P}_{\alpha}$. The completion of $\mathscr{P}_{\alpha}$ with respect to this norm is usually called the Rotation Algebra (it is a non-commutative analogue of 2-torus). Every representation of $\mathscr{P}_{\alpha}$ can be extended by continuity to a representation of $A_{\alpha}$, so in fact there is a natural one-one correspondence between representations of $\mathscr{P}_{\alpha}$ and $A_{\alpha}$.

A very important fact about the Rotation Algebra is that $A_{\alpha}$ is simple (i.e. does not contain any non-trivial 2-sided ideals) provided $\alpha \notin \mathbf{Q}$ (see e.g. [E-H, Pe, Po, Z]). In this case all its representations are faithful, hence isometric and preserve the spectrum ([D2]). This fact allowed us to define $A_{\alpha}$ in Sect. 2 as the norm closure of the Laurent polynomials in $U_{\alpha}, V_{\alpha}$ since we can consider a representation $\pi^{\prime \prime}: A_{\alpha} \rightarrow \mathscr{B}\left(l^{2}\left(\mathbf{Z}^{2}\right)\right)$ given by $\pi^{\prime \prime}(u)=U_{\alpha}, \pi^{\prime \prime}(v)=V_{\alpha}$.

Another representation of $A_{\alpha}$ is its representation $\pi^{\prime}$ in $l^{2}(\mathbf{Z})$ defined by the following operators $U, V$ :

$$
U \psi(n)=\psi(n+1), \quad V \psi(n)=e^{i(2 \pi \alpha n+\theta)} \psi(n),
$$

so that $\pi^{\prime}(u)=U, \pi^{\prime}(v)=V$.

We shall use the Rotation Algebra to prove the following well known

Theorem 4.2. For any $\alpha \notin \mathbf{Q}$ and any $\theta, \lambda \in \mathbf{R}$ spectra of $H_{\alpha, \theta, \lambda}$ and $\Delta_{\alpha, \lambda}$ coincide as subsets in $\mathbf{R}$.

Proof. $H_{\alpha, \theta, \lambda}$ and $\Delta_{\alpha, \lambda}$ are images of the same element

$$
h_{\alpha, \lambda}=u+u^{-1}+\lambda\left(v+v^{-1}\right) \in A_{\alpha},
$$

under different representations $\pi^{\prime}$ and $\pi^{\prime \prime}$ of $A_{\alpha}$. Hence the coincidence of spectra follows immediately from the preservation of spectra under faithful representations (in fact both these spectra coincide with the spectrum of $h_{\alpha, \lambda}$ in the algebra $A_{\alpha}$ itself).

Remark. A much more general connection between spectra of 1- and 2-dimensional discrete quasiperiodic operators follows from arguments given in [M-Z].

C. Now we shall prove the coincidence of IDS for AM and DML:

Theorem 4.3. If $\alpha \notin \mathbf{Q}$, then for all $\theta, \lambda, \mu \in \mathbf{R}$,

$$
N_{1}\left(\mu ; H_{\alpha, \theta, \lambda}\right)=N\left(\mu ; \Delta_{\alpha, \lambda}\right) .
$$


Proof. It is sufficient to prove the coincidence of the Laplace transforms, i.e. the equality

$$
M_{m}\left\{\left(e^{t H_{\alpha, \theta, \lambda}}\right)_{m, m}\right\}=\tau\left(e^{t \Delta_{\alpha, \lambda}}\right), \quad t \in \mathbf{C} .
$$

Consider the restriction of the trace $\tau$ to $A_{\alpha}\left(\right.$ in $\left.l^{2}\left(Z^{2}\right)\right)$. It will give us a trace on the abstract Rotation Algebra; this trace is norm continuous, hence uniquely defined by its values on $\mathscr{P}_{\alpha}$. Therefore it is uniquely defined by its values on all monomials of the form $u^{k} v^{l}, k, l \in \mathbf{Z}$. Obviously

$$
\tau\left(u^{k} v^{l}\right)=0 \quad \text { if } \quad|k|+|l| \neq 0, \quad \tau(1)=1 .
$$

Now this trace $\tau$ can be naturally transferred to all images of $A_{\alpha}$ under representations. We shall again denote the transferred traces by $\tau$. Obviously

$$
e^{t H_{\alpha, \theta, \lambda} \in \pi^{\prime}}\left(A_{\alpha}\right), \quad e^{t \Delta_{\alpha, \lambda} \in \pi^{\prime \prime}}\left(A_{\alpha}\right) .
$$

Hence to prove (4.6) it is sufficient to check that

$$
\tau(A)=M_{m}\left\{A_{m, m}\right\}, \quad A \in \pi^{\prime}\left(A_{\alpha}\right) .
$$

Here the right-hand side is norm continuous on $\pi^{\prime}\left(A_{\alpha}\right)$ since the norm convergence implies the uniform convergence of matrix elements. Due to (4.7) it is sufficient to check (4.8) for all operators of the form $A=U^{k} V^{l}$, where it is obvious. Note that the existence of the mean value in the right-hand side of (4.8) for all $A \in \pi^{\prime}\left(A_{\alpha}\right)$ follows from this argument as well.

Corollary 4.4. If $\alpha \notin \mathbf{Q}$ then the spectrum of $H_{\alpha, \theta, \lambda}$ coincides with the set of all points of growth for the corresponding IDS, i.e.

$$
\begin{aligned}
\operatorname{spec}\left(H_{\alpha, \theta, \lambda}\right)= & \left\{\mu \mid \mu \in \mathbf{R}, N_{1}\left(\mu+\varepsilon, H_{\alpha, \theta, \lambda}\right)\right. \\
& \left.-N_{1}\left(\mu-\varepsilon, H_{\alpha, \theta, \lambda}\right)>0 \text { for every } \varepsilon>0\right\} .
\end{aligned}
$$

Proof. Theorems 4.2 and 4.3 reduce this statement to the same one with $H_{\alpha, \theta, \lambda}$ replaced by $\Delta_{\alpha, \lambda}$ and $N_{1}\left(\mu ; H_{\alpha, \theta, \lambda}\right)$ replaced by $N\left(\mu ; \Delta_{\alpha, \lambda}\right)$. But then the conclusion is obvious due to the faithfulness of the trace $\tau$ (see Sect. 2).

Remark 4.5. Theorem 4.3 is true for $\alpha \in \mathbf{Q}$ and the proof works as well except (4.7) should be properly modified. But Theorem 4.2 and Corollary 4.4 are not true for $\alpha \in \mathbf{Q}$. Instead we have

$$
\operatorname{spec}\left(\Delta_{\alpha, \lambda}\right)=\bigcup_{\theta \in \mathbf{R}} \operatorname{spec}\left(H_{\alpha, \theta, \lambda}\right),
$$

so the right-hand side here should be put instead of the left-hand side in (4.9). The corresponding arguments can be found e.g. in [C-E-Y].

The result of Corollary 4.4 is of course well known and can be found in many sources in much more general situations (see e.g. [A-S, C-L, C-F-K-S, P-F, S2, S3]).

Remark 4.6. Let us consider a gauge transformation $T_{\gamma}: l^{2}\left(\mathbf{Z}^{2}\right) \rightarrow l^{2}\left(\mathbf{Z}^{2}\right)$ given for every $\gamma \in \mathbf{R}$ by the formula

$$
T_{\gamma} \psi(n)=e^{i \pi \gamma n_{1} n_{2}} \psi(n), \quad n=\left(n_{1}, n_{2}\right) \in \mathbf{Z}^{2} ; \quad \psi \in l^{2}\left(\mathbf{Z}^{2}\right) .
$$

Then

$$
T_{\gamma}^{-1} U_{\alpha} T_{\gamma}=U_{\alpha-\gamma}, \quad T_{\gamma}^{-1} V_{\alpha} T_{\gamma}=V_{\alpha+\gamma} .
$$


Therefore replacing $U_{\alpha}, V_{\alpha}$ by $U_{\alpha-\gamma}, V_{\alpha+\gamma}$ does not change the commutation relation (2.3) and gives again a representation of $A_{\alpha}$. In particular we can take $\gamma=\alpha$ to get the pair $U_{0}, V_{2 \alpha}$. The corresponding operator $\Delta_{\alpha, \lambda}=T_{\gamma}^{-1} \Delta_{\alpha, \lambda} T_{\gamma}=U_{0}+U_{0}^{*}$ $+\lambda\left(V_{2 \alpha}+V_{2 \alpha}^{*}\right)$ is exactly the operator considered in [B2, Su] and called the Harper operator there. It has the same spectrum and the same properties of eigenfunctions as $\Delta_{\alpha, \lambda}$.

\section{Gap Labelling and Ten Martini Problem}

The Gap Labelling theory is quite a developed field now, closely connected with $K$-theory of $C^{*}$-algebras (see e.g. an extensive review paper [B2]). We shall need a specific fact from this theory that we will formulate now.

For any *-algebra $A$ over $\mathbf{C}$ denote

$$
\operatorname{Proj}(A)=\left\{P \mid P \in A, P^{2}=P=P^{*}\right\},
$$

i.e. $\operatorname{Proj}(A)$ is the set of all self-adjoint projections in $A$ (if $A$ is a subalgebra in $\mathscr{B}(\mathscr{H}$ ) for a Hilbert pace $\mathscr{H}$, then $\operatorname{Proj}(A)$ is the set of all orthogonal projections in $A$ ). Now if $\tau: A \rightarrow \mathbf{C}$ is a trace on $A$ then $\tau(P)$ for a projection $P \in \operatorname{Proj}(A)$ can be considered as a dimension (of the image of $P$ in the case when $A \subset \mathscr{B}(\mathscr{H})$ ). So the set $\tau(\operatorname{Proj}(A))$ of all possible dimensions is important; it gives the first classification of factors - see e.g. [D1]. It follows from generalities on von Neumann algebras ([D1]) that $\tau\left(\operatorname{Proj}\left(W_{\alpha}\right)\right)=[0,1]$. Consider the rotation algebra $A_{\alpha} \subset W_{\alpha}$. Then the main result of the Gap Labelling theory in this case is:

$$
\tau\left(\operatorname{Proj}\left(A_{\alpha}\right)\right)=(\mathbf{Z}+\mathbf{Z} \alpha) \cap[0,1]
$$

(M. Rieffel [R], M. Pimsner and D. Voiculescu [P-V]).

For any self-adjoint operator $H \in \mathscr{B}(\mathscr{H})$ denote by $C^{*}(H)$ the $C^{*}$-algebra generated by $H$; this is the commutative algebra of all operators in $\mathscr{H}$ having the form $f(H)$, where $f \in C(\operatorname{spec}(H))$. In particular let $H=\int \mu d E_{\mu}$ be the spectral decomposition of $H$. Note that we always have $E_{\mu} \in W^{*}(H)$, where $W^{*}(H)$ is the von Neumann algebra generated by $H$, i.e. the set of all operators of the form $f(H)$, where $f$ is a bounded Borel function on $\operatorname{spec}(H)$.

Proposition 5.1. Suppose that $H$ has no point spectrum. Then $\mu$ is in the closure of a gap of the spectrum of $H$ (i.e. $\mu$ is in a gap or is an end of a gap) if and only if $E_{\mu} \in C^{*}(H)$.

Proof. By definition $E_{\mu} \in C^{*}(H)$ means that $E_{\mu}=f(H)$ for a continuous function $f: \operatorname{spec}(H) \rightarrow \mathbf{R}$. But this is the case exactly when $\mu$ is in a gap or is an end of a gap.

For the sake of brevity denote $N(\mu)=N\left(\mu ; \Delta_{\alpha, \lambda}\right)$ later in this section.

Corollary 5.2. If $\mu$ is in a gap of $\operatorname{spec}\left(\Delta_{\alpha, \lambda}\right)$ then $N(\mu) \in(\mathbf{Z}+\mathbf{Z} \alpha) \cap[0,1]$.

Proof. Obviously $C^{*}\left(\Delta_{\alpha, \lambda}\right) \subset A_{\alpha}$, so the statement follows from (5.1) and Proposition 5.1

Now the numbers in the countable set $(\mathbf{Z}+\mathbf{Z} \alpha) \cap[0,1]$ can be used as labels of gaps. Obviously labels of different gaps are different because of the faithfulness of the trace $\tau$. The important question is: which numbers in the set $(\mathbf{Z}+\mathbf{Z} \alpha) \cap[0,1]$ 
are really labels of the gaps, i.e. which ones are really values of $N(\mu)$ on gaps? Denote the set of all labels of (true) gaps by $\mathscr{L}_{\alpha, \lambda}$. Obviously $\mathscr{L}_{\alpha, \lambda} \subset$ $(\mathbf{Z}+\mathbf{Z} \alpha) \cap[0,1]$. Now the words of M. Kac "All gaps are there" (see Sect. 1) can be interpreted as the equality

$$
\mathscr{L}_{\alpha, \lambda}=(\mathbf{Z}+\mathbf{Z} \alpha) \cap[0,1],
$$

and STMP or Strong Ten Martini Problem is to establish that (5.2) is true for all $\alpha \notin \mathbf{Q}, \lambda \neq 0$.

Proposition 5.3. If (5.2) is true then $\operatorname{spec}\left(\Delta_{\alpha, \lambda}\right)=\operatorname{spec}\left(H_{\alpha, \theta, \lambda}\right)$ is a Cantor set (i.e. a compact nowhere dense set in $\mathbf{R}$ without isolated points).

Proof. The absence of isolated points in the spectrum is well known and also follows from Theorem 2.7. Hence if $\operatorname{spec}\left(\Delta_{\alpha, \lambda}\right)$ is not Cantor, then there exists a non-empty interval $(a, b) \subset \operatorname{spec}\left(\Delta_{\alpha, \lambda}\right) ; a<b$. Then the (non-empty) interval $(N(a)$, $N(b))$ contains a point $\xi \in(\mathbf{Z}+\mathbf{Z} \alpha) \cap[0,1]$. Since $N(\cdot)$ is continuous, there exists $\mu \in(a, b)$ such that $N(\mu)=\xi$. Hence $\xi \notin \mathscr{L}_{\alpha, \lambda}$ which contradicts (5.2).

Note now that for any $\mathrm{II}_{1}$-factor $W$ (with the trace $\tau: W \rightarrow \mathbf{C}$ ) and any two projections $P, Q \in \operatorname{Proj}(W)$ the equality $\tau(P)=\tau(Q)$ is equivalent to the existence of a unitary $U \in W$ such that $U^{-1} P U=Q$ (i.e. to the fact that $P$ and $Q$ are unitary equivalent in $W$ ). This motivates the introduction of the following inclusion:

$$
\left[U^{-1}\left(\operatorname{Proj}\left(A_{\alpha}\right)\right) U\right] \cap W^{*}\left(\Delta_{\alpha, \lambda}\right) \subset C^{*}\left(\Delta_{\alpha, \lambda}\right) .
$$

Now let us formulate the Superstrong Ten Martini Problem (SSTMP): prove or disprove the following conjecture:

The inclusion (5.3) is true for all $\alpha \notin \mathbf{Q}, \lambda \neq 0$ and every unitary $U \in W_{\alpha}$.

Proposition 5.4. The positive solution of SSTMP implies the positive solution of STMP. More exactly, if for some $\alpha, \lambda$ the inclusion of (5.3) is true for all unitary $U \in W_{\alpha}$, then (5.2) is true for the same $\alpha, \lambda$.

Proof. Suppose that (5.3) is true for any unitary $U \in W_{\alpha}$. Consider then any $\mu \in \mathbf{R}$ with $N(\mu) \in \mathbf{Z}+\mathbf{Z} \alpha$. (Recall that $N(\mu) \in[0,1]$ automatically.) Then for the corresponding spectral projection $E_{\mu}$ (of $\Delta_{\alpha, \lambda}$ ) there exists a unitary operator $U \in W_{\alpha}$ such that $U^{-1} E_{\mu} U \in A_{\alpha}$. Indeed (5.1) implies that the algebra $A_{\alpha}$ contains a projection $P$ with the same trace $\tau(P)=\tau\left(E_{\mu}\right)$, so we can apply the argument given before (5.3). Therefore $E_{\mu}$ belongs to the set in the left-hand side of (5.3). Hence $E_{\mu} \in C^{*}\left(\Delta_{\alpha, \lambda}\right)$ and $\mu$ is in a closure of a gap due to Proposition 5.1. This proves (5.2).

The motivation of (5.3) is obvious for the case $U=I$. In this case all elements in $A_{\alpha}$ are norm limits of polynomials in $U_{\alpha}, V_{\alpha}$. If at the same time such an element is in $W^{*}\left(\Delta_{\alpha, \lambda}\right)$, i.e. if it is a weak limit of polynomials in $\Delta_{\alpha, \lambda}$, then it is natural to expect that it is really a norm limit of such polynomials.

On the other hand note that (5.3) is not true if $\lambda=0$; so non-commutativity should play an important role here.

Acknowledgements. As one of the source of my inspiration I should mention beautiful lectures of J. Bellissard in Nantes Summer School (1991). Then during my visit to MIT in the Fall of 1991 I enjoyed V. Guillemin seminar where he made in particular a good publicity of a remarkable paper by M.-D. Choi, G. Elliott and N. Yui [C-E-Y]. I am also grateful to G. Elliott, J. Sjöstrand and T. Sunada for useful discussions. 


\section{References}

[A - A] Aubry, S., André, G.: Analyticity breaking and the Anderson localization in incommensurate lattices. Ann. Israel Phys. Soc. 3, 133-164 (1980)

[A-M-S] Avron, J.E., van Mouche, P., Simon, B.: On the measure of the spectrum for the almost Mathieu operator. Commun. Math. Phys., 132, 103-118 (1990)

[A-S] Avron, J.E., Simon, B.: Almost periodic Schrödinger operators, II. The density of states. Duke Math. J. 50, 369-391 (1983)

[A] Ya. Azbel.: Energy spectrum of a conduction electron in a magnetic field. Soviet Phys. JETP 19:3 (1964)

[B1] Bellissard, J.: Almost periodicity in solid state physics and $C^{*}$-algebras. In: C. Berg. B. Fuglede (eds.) The Harald Bohr Centenary, Mat.-Fys. Medd. Danske Vid. Selsk., 42:3, 35-75 (1989)

[B2] Bellissard, J.: Gap labelling theorems for Schrödinger operators. In: M. Waldschmidt (eds) From Number Theory to Physics, Berlin, Heidelberg, New York: Springer, 1992 pp. 538-630

[B3] Bellissard, J.: Le papillon de Hofstadter [d'après B. Helffer et J. Sjöstrand]. Séminaire Bourbaki, 1991-92, n 745, Novembre 1991

[B-L-T1] Bellissard, J., Lima, R., Testard, D.: A metal-insulator transition for the almost Mathieu operator. Commun. Math. Phys. 88, 207-234 (1983)

[B-L-T2] Bellissard, J., Lima, R., Testard, D.: Almost Periodic Schrödinger Operators. In: Mathematics + Physics. Lectures on recent results. Vol. 1, L. Streit (ed.), Singapore, Philadelphia; World Sci. Pub., 1985, pp. 1-64

[B-S] Bellissard, J., Simon, B.: Cantor spectrum for the almost Mathieu equation. J. Funct. Anal. 48, 408-419 (1982)

[B-F] Buslaev, B., Fedotov, A.: Complex WKB method for Harper's equation. Institut Mittag-Leffler, Report No. 11, 1992/93

[C-L] Carmona, R., Lacroix, J.: Spectral theory of random Schrödinger operators. Boston: Birkhäuser, 1990

[Ch] Chambers, W.: Linear network model for magnetic breakdown in two dimensions. Phys. Rev. A140, 135-143 (1965)

[C-E-Y] Choi, M.-D., Elliott, G., Yui, N.: Gauss polynomials and the rotation algebra. Invent. Math. 99, 225-246 (1990)

[C] Chulaevsky, V.A.: Almost periodic operators and related non-linear integrable systems. Manchester: Manchester Univ. Press, 1989

[C-D] Chulaevsky, V.A., Delyon, F.: Purely absolutely continuous spectrum for almost Mathieu operators. J. Stat. Phys. 55, 1279-1284 (1989)

[C-S] Craig, W., Simon, B.: Log Hölder continuity of the integrated density of states for stochastic Jacobi matrices. Commun. Math. Phys. 90, 207-218 (1983)

[C-F-K-S] Cycon, H.L., Froese, R.G., Kirsch, W., Simon, B.: Schrödinger operators. Berlin, Heidelberg, New York: Springer, 1987

[D] Delyon, F.: Absence of localization for the almost Mathieu equation. J. Phys. A20, L21-L23 (1987)

[D-S] Delyon, F., Souillard, B.: Remark on the continuity of the density of states of ergodic finite difference operators. Commun. Math. Phys., 94, 289-291 (1984)

[D1] Dixmier, J.: Von Neumann algebras. Amsterdam; North-Holland, 1981

[D2] Diximier, J.: $C^{*}$-algebras. Amsterdam: North-Holland, 1982

[E-H] Effros, E.G., Hahn, F.: Locally compact transformation groups and $C^{*}$-algebras. Mem. Am. Math. Soc. 75, (1967)

[E1] Eilliott, G.A.: Gaps in the spectrum of an almost periodic Schrödinger operator. C.R. Math. Rep. Acad. Sci. Canada 4, 255-259 (1982)

[E2] Eilliott, G.A.: Gaps in the spectrum of an almost periodic Schrödinger operator. II. In: H. Araki, E.G. Effros (eds.), Geometric Methods in Operator Algebras. Pitman Research Notes in Math. Series 123, London: Longman, 1986 pp. 181-191

[F-P] Figotin, A., Pastur, L.: The positivity of Lyapunov exponent and absence of absolutely continuous spectrum for almost Mathieu equation. J. Math. Phys. 25, 774-777 (1984) 
[F-S-W] Fröhlich, J., Spencer, T., Witwer, P.: Localization for a class of one-dimensional quasi-periodic Schrödinger operators. Commun. Math. Phys. 132, 5-25 (1990)

[G] Gusev, A.I.: The density of states and other spectral invariants of self-adjoint elliptic operators with random coefficients. Math. USSR Sbornik 33, 185-202 (1977)

[Ha] Harper, P.G.: Single band motion of conduction electrons in a uniform magnetic field. Proc. Phys. Soc. London A68, 874-892 (1955)

[H-K-S] Helffer, B., Kerdelhué, P., J. Sjöstrand: Le papillon de Hofstadter revisité. Mémoires de la S.M.F., no. 43, 118:3, 1-87 (1990)

[H-S1] Helffer, B., Sjöstrand, J.: Analyse semi-classique pour l'équation de Harper (avec application à l'équation de Schrödinger avec champ magnetique). Mémoires de la S.M.F., no. 34, 116:4, 1-113 (1988)

[H-S2] Helffer, B., Sjöstrand, J.: Analyse semi-classique pour l'équation de Harper II. Comportement semi-classique près d'un rationnel. Mémoires de la S.M.F., no. 40, 118, 1-139 (1990)

[H-S3] Helffer, B., Sjöstrand, J.: Semi-classical analysis for Harper's equation III. Cantor structure of the spectrum. Mémoires de la S.M.F., no. 39, 117:4, 1-124 (1989)

[Ho] Hofstadter, D.: Energy levels and wave functions of Bloch electrons in rational and irrational magnetic fields. Phys. Rev. B 14, 2239-2249 (1976)

[J] Jitomirskaya, S.Ya.: Anderson localization for the almost Mathieu equation: A nonperturbative proof, Commun. Math. Phys., to appear

[J-M] Johnson, R., Moser, J.: The rotation number for almost periodic potentials. Commun. Math. Phys. 84, 403-438 (1982)

[L1] Last, Y.: A relation between a.c. spectrum of ergodic Jacobi matrices and the spectra of periodic approximants. Commun. Math. Phys. 151, 183-192 (1993)

[L2] Last, Y.: Zero measure spectrum for the almost Mathieu operator. Preprint, January 1993

[L-W] Last, Y., Wilkinson, M.: A sum rule for the dispersion relations of the rational Harper's equation. J. Phys. A25, 6123-6133 (1992)

[M-Z] Mandelshtam, V.A., Zhitomirskaya, S.Ya.: 1D-quadiperiodic operators. Latent symmetries. Commun. Math. Phys. 139, 589-604 (1991)

[Mo] van Mouche, P.: The coexistence problem for the discrete Mathieu operator. Commun. Math. Phys. 122, 23-24 (1989)

[Mu] Murphy, G.J.: $C^{*}$-algebras and operator theory. Boston e.a.: Academic Press, 1990

[N] Novikov, S.P.: Two dimensional operators in periodic fields. J. Soviet Math., 28:1, 1-20 (1985)

[Pa] Pastur, L.A.: Spectra of random self-adjoint operators. Russ. Math. Surv. 28, 1-67 (1973)

[P-F] Pastur, L., Figotin, A.: Spectra of random and almost periodic operators. Berlin, Heidelberg, New York: Springer, 1992

[Pe] Pedersen, G.K.: $C^{*}$-algebras and their automorphism groups. London: Academic Press, 1979

[P] Peierls, R.: Zur Theorie des Diamagnetismus von Leitungselektronen. Z. Phys. 80, 763-791 (1933)

[P-V] Pimsner, M., Voiculescu, D.: Imbedding the irrational rotation $C^{*}$-algebra into a $A F$ algebra. J. Oper. Theory 4, 201-210 (1980)

[Po] Power, S.C.: Simplicity of $C^{*}$-algebras of minimal dynamical systems. J. Lond. Math. Soc. 18, 534-538 (1978)

[R1] Riedel, N.: Point spectrum for the almost Mathieu equation. C.R. Math. Rep. Acad. Sci. Canada, Vol. VIII, No. 6, 399-403 (1986)

[R2] Riedel, N.: Almost Mathieu operators and rotation $C^{*}$-algebras. Proc. Lond. Math. Soc. 56:3, 281-302 (1988)

[R3] Riedel, N.: On spectral properties of almost Mathieu operators and connections with irrational rotation $C^{*}$-algebras. Rocky Mountain J. of Math. 20:2, 539-548 (1990)

[R4] Riedel, N.: Spectra and resolvents of certain polynomials in the irrational rotation algebra. Indiana Univ. Math. J. 39:4, 937-945 (1990)

[R5] Riedel, N.: The spectrum of a class of almost periodic operators. Preprint 1991; revised version 1993

[R6] Riedel, N.: Absence of Cantor spectrum for a class of Schrödinger operators. Bull. Am. Math. Soc. 29:1, 85-87 (1993) 
[R7] Riedel, N.: Regularity of the spectrum for the Almost Mathieu operator. Preprint, 1993

[R] Rieffel, M.: $C^{*}$-algebras associated with irrational rotations. Pac. J. Math., 93:2, 415-419 (1981)

[S1] Shubin, M.A.: Almost periodic pseudo-differential operators and von Neumann algebras. Trudy Mosov. Mat. Obsc. 35, 103-164 (1976). English translation: Trans. Moscow Math. Soc., Issue 1, 103-166 (1979)

[S2] Shubin, M.A.: The density of states of selfadjoint elliptic operators with almost periodic coefficients. Trudy Sem. Petrovsk. 3, 243-275 (1978). English translation: Amer. Math. Soc. Transl. (2) Vol. 118, 307-339 (1982)

[S3] Shubin, M.A.: The spectral theory and the index of elliptic operators with almost periodic coefficients. Russ. Math. Surv. 34:2, 109-157 (1979)

[S] Simon, B.: Almost periodic Schrödinger operators: A review. Adv. in Appl. Math. 3, 463-490 (1982)

[Si] Sinai, Ya. G.: Anderson localization for one-dimensional difference Schrödinger operator with quasiperiodic potential. J. Stat. Phys. 46, 861-909 (1987)

[S1] Slivnyak, I.M.: Spectrum of the Schrödinger operator with random potential. Zh. Vychisl. Mat. i Mat. Fiz. 6:6, 1104-1108 (1966)

[Su] Sunada, T.: Generalized Harper operator on a graph. Workshop on Zeta Functions in Number Theory and Geometric Analysis in Honor of Jun-Ichi Igusa, John Hopkins University, March 29-April 3, 1993

[T1] Thouless, D.J.: Bandwidth for a quadiperiodic tight binding model. Phys. Rev. B28, 4272-4276 (1983)

[T2] Thouless, D.J.: Scaling for the discrete Mathieu equation. Commun. Math. Phys. 127, 187-193 (1990)

[T-T1] Thouless, D.J., Tan, Y.: Scaling, localization and bandwidths for equations with competing periods. Physica A177, 567-577 (1991)

[T-T2] Thouless, D.J., Tan, Y.: Total bandwidth for the Harper equation III. Corrections to scaling. J. Phys. A24, 4055-4066 (1991)

[Wa] Watson, G.I.: WKB analysis of energy band structure of modulated systems. J. Phys. A24, 4999-5010 (1991)

[W] Wilkinson, M.: Critical properties of electron eigenstates in incommensurate systems. Proc. Royal Soc. Lond. A391, 305-330 (1984)

[Z] Zeller-Meier, G.: Produit croisés d'une $C^{*}$-algebre par une groupe d'automorphismes. J. Math. Pures et Appl. 47, 101-239 (1968) 
